\title{
ASPECTOS CLÍNICO-EPIDEMIOLÓGICOS DA SÍFILIS GESTACIONAL NO NORDESTE DO BRASIL
}

Clinical-epidemiological aspects of gestational syphilis in northeast Brazil

Aspectos clínico-epidemiológicos de la sífilis gestacional en el noreste de Brasil

Sandy Soares de Sousa • Acadêmica do curso de Enfermagem pela Universidade Estadual do Piauí (UESPI) • Campus Doutora Josefina Demes • E-mail: soaressandy21@gmail.com

Yanneck Barbosa Silva • Acadêmica do curso de Enfermagem pela UESPI • Campus Doutora Josefina Demes • E-mail: yannecksilva@hotmail.com Iara Maria Lima da Silva • Acadêmica do curso de Enfermagem pela UESPI • Campus Doutora Josefina Demes • E-mail: iara-raiure01@hotmail.com

Hernandes Flanklin Carvalho Oliveira - Acadêmico do curso de Enfermagem pela UESPI • Campus Doutora Josefina Demes • E-mail: franklin.oliveira@yahoo.com Antonio Gabriel dos Santos Castro - Acadêmico do curso de Enfermagem pela UESPI • Campus Doutora Josefina Demes • E-mail: gabrielcastroenf@gmail.com Augusto Cezar Antunes de Araújo Filho • Professor Doutor da Universidade Estadual do Piauí • Campus Doutora Josefina Demes • E-mail: augustoantunes@frn.uespi.br

Autor correspondente:

Augusto Cezar Antunes de Araújo Filho• E-mail: augustoantunes@frn.uespi.br 


\section{RESUMO}

Introdução: A sífilis é uma das infecções transmissíveis de maior impacto global. Quando acomete em gestante é denominada sífilis gestacional, que pode ocasionar resultados fetais graves e também a sífilis congênita, que tem sido uma das principais responsáveis pela alta carga de morbimortalidade de recém-nascidos. Por conta disso, a sífilis gestacional tem atraído ações mundiais em prol do seu combate, porém, apesar dos esforços, há um aumento persistente, ano após ano dessa infecção, principalmente no Nordeste brasileiro. Objetivo: Descrever o perfil epidemiológico da sífilis gestacional no Nordeste brasileiro, entre os anos de 2014 e 2018. Metodologia: Estudo descritivo, transversal, realizado por meio de dados secundários de casos confirmados de sífilis em gestante entre os anos de 2014 a 2018, em todos os Estados do Nordeste brasileiro. As variáveis investigadas são referentes ao perfil sociodemográfico materno (idade, escolaridade e cor/raça), à idade gestacional e à classificação clínica da sífilis. Resultados: No período investigado foram notificados 41.605 casos de sífilis em gestantes, com aumento expressivo de quase 287\% do ano de 2014 (5.137 casos) para 2018 (14.507 casos). Entre os Estados nordestinos, os números foram mais alarmantes, respectivamente, em: Bahia, Pernambuco, Ceará e Maranhão. Observou-se, entre os casos, predomínio de mães na faixa etária de 20 a 29 anos, com ensino fundamental incompleto, de raça parda e diagnosticadas no $3^{\circ}$ trimestre com sífilis primária. Conclusões: $O$ estudo demonstrou que os casos de sífilis cresceram gradativamente, o que indica a necessidade de intervenções precoces tanto no rastreio como no tratamento das gestantes e dos seus parceiros, a fim de minimizar os casos de sífilis congênita.

Palavras-Chave: Sífilis. Gestantes. Perfil de Saúde. Saúde Materna.

\section{ABSTRACT}

Introduction: Syphilis is one of the most globally impacted communicable infections. When it affects a pregnant woman, it is called gestational syphilis, which can cause serious fetal results and also congenital syphilis, which has been one of the main factors responsible for the high burden of morbidity and mortality of newborns. Because of this, gestational syphilis has attracted worldwide actions in order to fight it, however, despite efforts, there is a persistent increase, year after year, of this infection, mainly in Northeast Brazil. Objective: To describe the epidemiological profile of gestational syphilis in Northeastern Brazil, between the years 2014 and 2018. Methodology: Descriptive, cross-sectional study, carried out using secondary data from confirmed cases of syphilis in pregnant women between the years 2014 to 2018, in all states in the Northeast of Brazil. The investigated variables refer to the maternal sociodemographic profile (age, education and color / race), gestational age and the clinical classification of syphilis. Results: During the investigated period 1,605 cases of syphilis were reported in pregnant women, with a significant increase almost 287\% from 2014 (5,137 cases) to 2018 (14,507 cases). Among the rtheastern states, the numbers were more alarming, respectively, in: Bahia, nambuco, Ceará and Maranhão. Among the cases, there was a predominance of thers aged 20 to 29 years, with incomplete elementary education, of mixed race diagnosed in the 3rd trimester with primary syphilis. Conclusions: The study 
demonstrated that the cases of syphilis grew gradually, which indicates the need for early interventions both in the screening and in the treatment of pregnant women and their partners, in order to minimize the cases of congenital syphilis.

Keywords: Syphilis. Pregnant Women. Health Profile. Maternal Health.

\section{RESUMEN}

Introducción: La sífilis es una de las infecciones transmisibles con mayor impacto a nivel mundial. Cuando afecta a una mujer embarazada se le llama sífilis gestacional, que puede ocasionar severos resultados fetales y también sífilis congénita, que ha sido uno de los principales factores responsables de la alta carga de morbilidad y mortalidad de los recién nacidos. Debido a esto, la sífilis gestacional ha atraído acciones a nivel mundial para combatirla, sin embargo, a pesar de los esfuerzos, hay un aumento persistente, año tras año, de esta infección, principalmente en el noreste de Brasil. Objetivo: Describir el perfil epidemiológico de la sífilis gestacional en el noreste de Brasil, entre los años 2014 y 2018. Metodología: Estudio descriptivo, transversal, realizado con datos secundarios de casos confirmados de sífilis en gestantes entre los años 2014 a 2018, en todos los estados del Nordeste de Brasil. Las variables investigadas están relacionadas con el perfil sociodemográfico materno (edad, educación y color / raza), la edad gestacional y la clasificación clínica de la sífilis. Resultados: En el período investigado se reportaron 41.605 casos de sífilis en mujeres embarazadas, con un incremento significativo de casi $287 \%$ desde 2014 (5.137 casos) hasta 2018 (14.507 casos). Entre los estados del noreste, las cifras fueron más alarmantes, respectivamente, en: Bahía, Pernambuco, Ceará y Maranhão. Entre los casos, hubo predominio de madres de 20 a 29 años, con educación primaria incompleta, raza morena y diagnosticadas en el 3er trimestre de sífilis primaria. Conclusiones: El estudio demostró que los casos de sífilis crecieron paulatinamente, lo que indica la necesidad de intervenciones tempranas tanto en el cribado como en el tratamiento de la gestante y sus parejas, con el fin de minimizar los casos de sífilis congénita.

Palabras clave: Ś́filis. Mujeres Embarazadas. Perfil de Salud. Salud Materna. 


\section{ciênncia
pural}

\section{Introdução}

A sífilis é uma das infecções sexualmente transmissíveis (IST) de maior impacto global ${ }^{1}$. Quando acomete gestantes, é denominada de sífilis gestacional (SG), a qual, sem tratamento ou com tratamento inadequado, pode ser transmitida ao feto em qualquer estágio da gestação². No que se refere às manifestações clínicas, a sífilis gestacional apresenta semelhança às da sífilis adquirida, a qual acomete a população geral. A infecção por sífilis é dividida em estágios fundamentados em achados clínicos (primária, secundária, latente recente, latente tardia e terciária) ${ }^{3}$.

Em 2008, a Organização Mundial da Saúde (OMS) estimou que 1,86 milhão de casos de sífilis ocorrem globalmente entre mulheres grávidas a cada ano e que uma grande proporção delas não são tratadas ou recebem tratamento inadequado ${ }^{4}$. Em 2018, no Brasil, a taxa de detecção de SG foi de 21,4 casos por 1000 nascidos vivos¹.

A sífilis gestacional pode causar resultados fetais graves, incluindo morte fetal e neonatal precoce, natimorto, prematuridade e baixo peso ao nascer, bem como na sífilis congênita $(S C)^{2}$. No cenário recente, a SC tem sido uma das principais responsáveis pela alta carga de morbimortalidade entre recém-nascidos. Globalmente, é a principal causa de mais de 212.000 mortes fetais por ano. Os dados devem ser encarados como evitáveis, visto que apesar de grave, a SC pode ser combatida com a detecção eficaz da SG e tratamento das infectadas e seus parceiros, desta forma é possível impedir que transmissão ao feto aconteça ${ }^{5}$.

Por conta disso, a SG tem atraído ações mundiais em prol do seu combate. Porém, apesar dos esforços, há um aumento global persistente, ano após ano, na prevalência dessa infecção em gestantes. O Nordeste brasileiro merece grande atenção, tendo em vista que, no período entre os anos de 2005 e junho de 2019, ocupou o segundo lugar entre as regiões brasileiras com mais casos de SG tificados, com $21 \%$ das 324.321 notificações de sífilis em gestantes. Ademais, em 18 observou-se aumento de 59,6\% no número de notificações na Região Nordeste1.

Diante da perspectiva de aumento das taxas de detecção da SG, no Brasil e, retudo, no Nordeste, e por conseguinte, das taxas de SC, este estudo tem por 
objetivo descrever o perfil epidemiológico da sífilis gestacional no Nordeste brasileiro, entre os anos de 2014 e 2018.

\section{Metodologia}

Estudo descritivo, transversal, realizado por meio de dados secundários de casos confirmados de sífilis em gestante entre os anos de 2014 e 2018, em todos os Estados do Nordeste brasileiro. A região Nordeste é composta por nove Estados: Alagoas, Bahia, Ceará, Maranhão, Paraíba, Pernambuco, Piauí, Rio Grande do Norte e Sergipe.

A população do estudo foi composta por todos os casos notificados de sífilis em gestante no Nordeste brasileiro, no período entre 2014 e 2018 ( $n=41.605)$. Os dados são oriundos do Sistema de Informação de Agravos de Notificação (SINAN), os quais estão disponíveis de forma online e gratuita no site do Departamento de Informática do Sistema Único de Saúde (DATASUS). Ressalta-se que a escolha do período se deu por conta da incompletude dos dados no ano de 2019.

Os dados foram extraídos em junho de 2020, e, após a extração, foram agrupados e analisados no Microsoft Excel ${ }^{\circledR}$, software em que foi realizada a análise estatística descritiva (frequência absoluta e relativa). As variáveis investigadas são referentes ao perfil sociodemográfico materno (idade, escolaridade e cor/raça), à idade gestacional e à classificação clínica da sífilis. Além disso, foram analisados os casos notificados e as taxas de detecção por ano e por Estado do Nordeste brasileiro.

Tendo em vista que este estudo fez uso de dados secundários, que estão disponíveis de forma online e gratuita, não foi necessária a submissão ao Comitê de Ética em Pesquisa, levando em consideração a resolução $n^{\circ}$. 510, de 07 de abril de 2016, do Conselho Nacional de Saúde. 


\section{Resultados}

No período investigado foram notificados 41.605 casos de sífilis em gestantes. Verifica-se aumento expressivo no número de casos entre os anos, em que, no ano de 2014, foram notificados 5.137 casos e, em 2018, 14.705 registros.

Gráfico 1. Casos notificados de sífilis em gestantes no Nordeste brasileiro. 2014-2018. Teresina-PI, 2020.

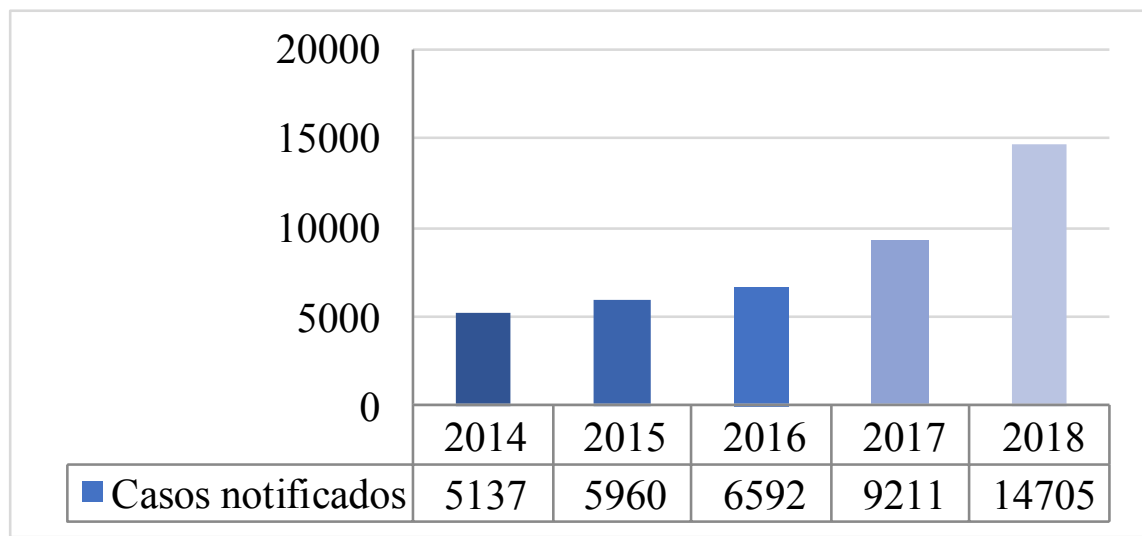

Observa-se, no gráfico 2, que os Estados da Bahia, Pernambuco, Ceará e Maranhão registraram números de casos maiores que os demais Estados nordestinos. A Bahia, por sua vez, assumiu a primeira posição de casos notificados em todo o período investigado. 


\section{ciência 0100}

Gráfico 2. Casos notificados de sífilis em gestantes por Estado nordestino. 2014-2018. Teresina-PI, 2020.

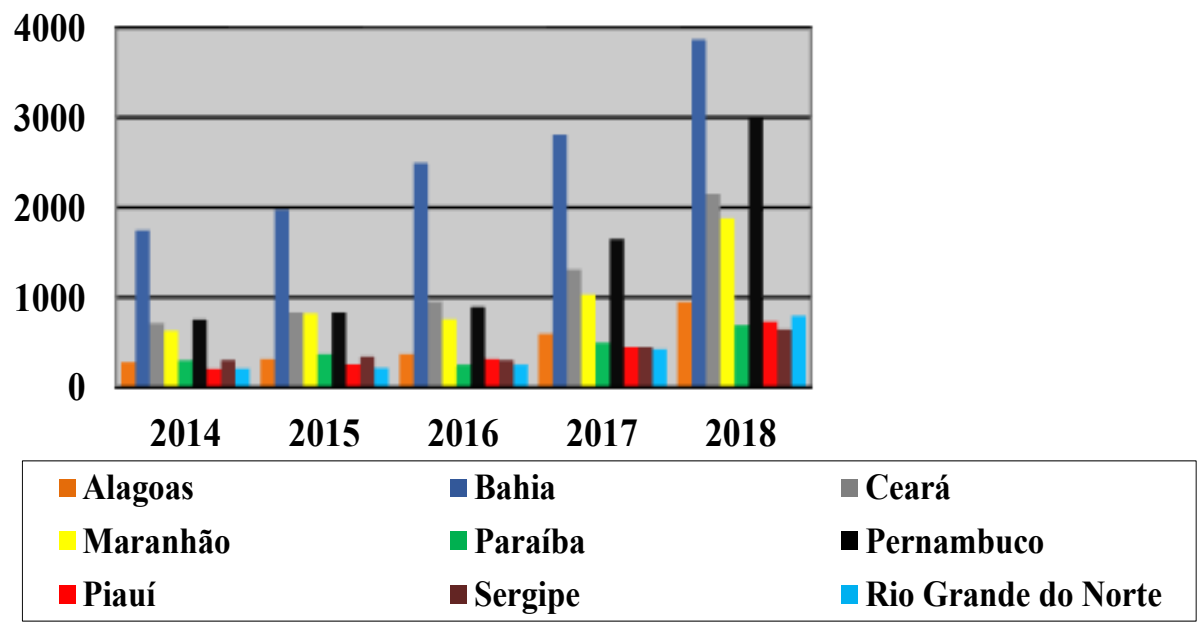

Durante todo o período investigado, os Estados da Bahia e de Sergipe, e, a partir de 2017, os de Alagoas e Pernambuco apresentaram taxas de detecção maiores que a média nordestina. 
Gráfico 3. Taxas de detecção (por 1.000 nascidos vivos) na Região Nordeste e por Estado nordestino. Teresina-PI, 2020.

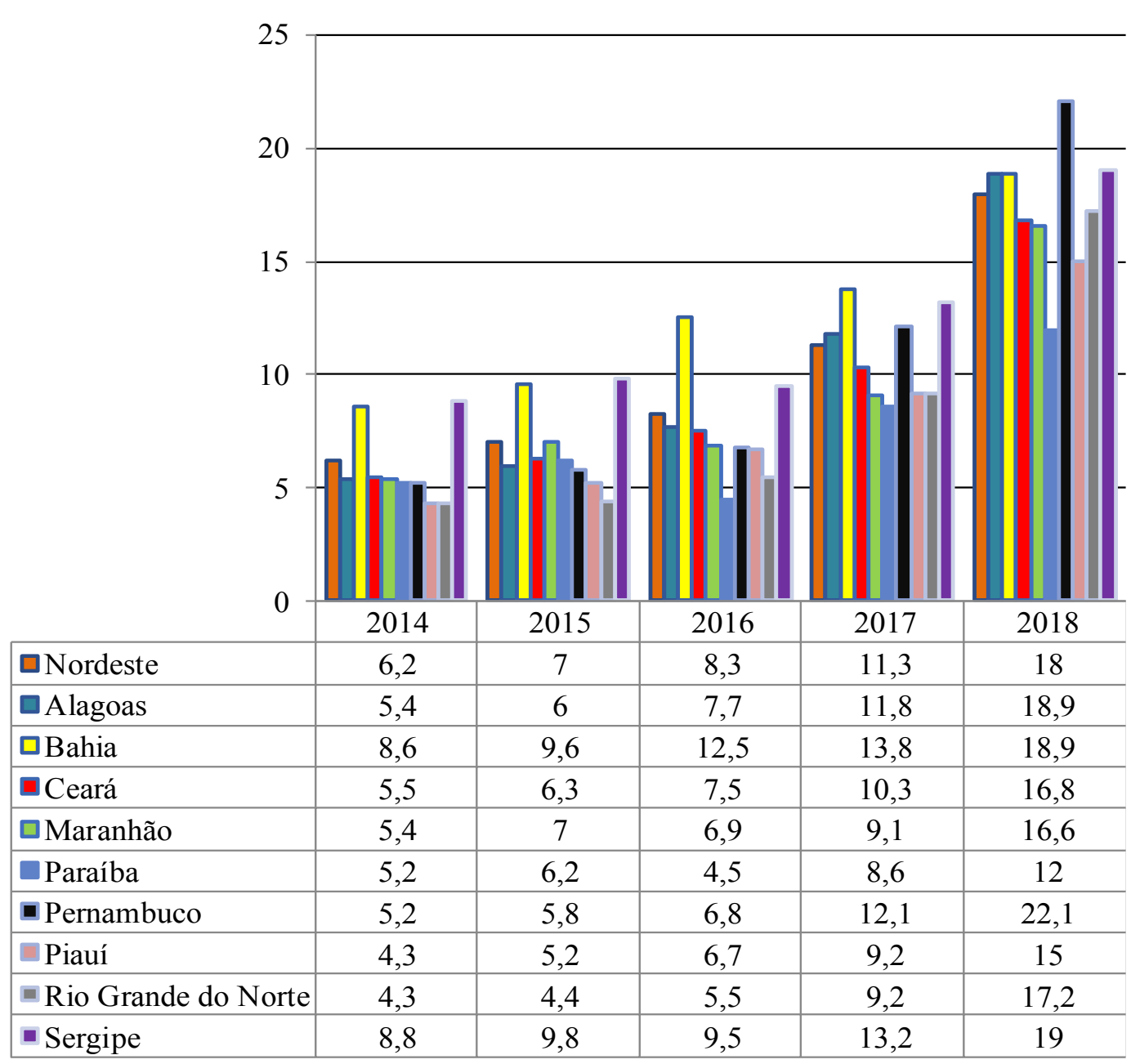

Verifica-se na Tabela 1, predomínio de mães na faixa etária de 20 a 29 anos, com ensino fundamental incompleto, de raça parda e diagnosticadas no $3^{\circ}$ trimestre com sífilis primária. 
Tabela 1. Características sociodemográficas das gestantes da região Nordeste. Teresina-PI, 2020.

\begin{tabular}{|c|c|c|c|c|c|c|c|c|c|c|c|c|}
\hline \multirow[t]{2}{*}{ Variável } & \multicolumn{2}{|c|}{2014} & \multicolumn{2}{|c|}{2015} & \multicolumn{2}{|c|}{2016} & \multicolumn{2}{|c|}{2017} & \multicolumn{2}{|c|}{2018} & \multicolumn{2}{|c|}{ Total } \\
\hline & $\mathbf{n}$ & $\%$ & $\mathbf{n}$ & $\%$ & $\mathbf{n}$ & $\%$ & $\mathbf{n}$ & $\%$ & $\mathbf{n}$ & $\%$ & $\mathbf{n}$ & $\%$ \\
\hline \multicolumn{13}{|l|}{ Faixa etária } \\
\hline 10 a 14 anos & 89 & 1,73 & 100 & 1,68 & 98 & 1,49 & 159 & 1,73 & 183 & 1,24 & 629 & 1,51 \\
\hline \multirow{2}{*}{15 a 19 anos } & 1.33 & 26,0 & 1.47 & 24,7 & 1.73 & 26,3 & 2.33 & 25,4 & \multirow{2}{*}{3.628} & 24,6 & 1051 & 25,2 \\
\hline & 9 & 8 & 2 & 0 & 4 & 2 & 9 & 0 & & 8 & 2 & 7 \\
\hline \multirow{2}{*}{20 a 29 anos } & 2.54 & 49,5 & 3.06 & 51,4 & 3.34 & 50,8 & 4.78 & 52,0 & \multirow{2}{*}{7.606} & 51,7 & 2135 & 51,3 \\
\hline & 3 & 2 & 5 & 3 & 7 & 0 & 9 & 0 & & 3 & 0 & 3 \\
\hline \multirow{2}{*}{30 a 39 anos } & 1.06 & 207 & 1.18 & 19,8 & 1.26 & 19,2 & 1.74 & 18,9 & \multirow{2}{*}{2.959} & 20,1 & \multirow{2}{*}{8218} & 19,7 \\
\hline & 3 & 20,1 & 5 & 8 & 9 & 6 & 2 & 2 & & 3 & & 6 \\
\hline $\begin{array}{c}40 \text { anos ou } \\
\text { mais }\end{array}$ & 101 & 1,97 & 138 & 2,31 & 140 & 2,13 & 180 & 1,95 & 327 & 2,22 & 886 & 2,13 \\
\hline \multicolumn{13}{|l|}{$\begin{array}{c}\text { Escolaridad } \\
\text { e }\end{array}$} \\
\hline Analfabeto & 109 & 2,12 & 95 & 1,59 & 90 & 1,37 & 103 & 1,12 & 131 & 0,89 & 528 & 1,27 \\
\hline Fundament & 1.92 & 37,5 & 2.19 & 36,7 & 2.32 & 35,3 & 3.15 & 34,2 & \multirow{2}{*}{4.956} & 33,7 & 14.55 & 34,9 \\
\hline Incompleto & 8 & 3 & 1 & 6 & 8 & 2 & 1 & 1 & & 0 & 4 & 8 \\
\hline \multicolumn{13}{|l|}{ Fundament } \\
\hline \multicolumn{13}{|l|}{ Completo } \\
\hline Médio & 504 & 9 81 & 640 & 10,8 & 734 & 11,1 & 1.14 & 12,4 & \multirow{2}{*}{1.822} & 12,3 & \multirow{2}{*}{4.856} & 11,6 \\
\hline Incompleto & 504 & & & 9 & 04 & 3 & 7 & 5 & & 9 & & 7 \\
\hline Médio & \multirow{2}{*}{654} & 12,7 & 712 & 11,9 & 32 & 12,6 & 1.43 & 15,5 & \multirow{2}{*}{2.586} & 17,5 & \multirow{2}{*}{6.218} & 14,9 \\
\hline Completo & & 3 & 112 & 5 & 032 & 2 & 4 & 7 & & 9 & & 5 \\
\hline $\begin{array}{c}\text { Superior } \\
\text { Incompleto }\end{array}$ & 30 & 0,58 & 51 & 0,85 & 45 & 0,68 & 90 & 0,98 & 131 & 0,89 & 347 & 0,83 \\
\hline $\begin{array}{l}\text { Superior } \\
\text { Completo }\end{array}$ & 28 & 0,55 & 28 & 0,47 & 42 & 0,64 & 67 & 0,73 & 135 & 0,92 & 300 & 0,72 \\
\hline $\begin{array}{c}\text { Não se } \\
\text { aplica }\end{array}$ & 2 & 0,04 & - & - & 3 & 0,04 & 2 & 0,02 & 2 & 0,01 & 9 & 0,02 \\
\hline Ignor & 1.57 & 30,7 & 1.75 & 25 & 1.96 & 29,8 & 2.43 & 26,4 & & 25,2 & 11.45 & 27,5 \\
\hline & 8 & 2 & 8 & & 5 & 1 & 9 & 8 & 712 & 4 & 2 & 3 \\
\hline
\end{tabular}

\section{Raça/Cor}

$\begin{array}{ccccccccccccc}\text { Branca } & 552 & 10,7 & 619 & 10,3 & 686 & 10,4 & 1.00 & 10,8 & 1.600 & 10,8 & 4.457 & 10,7 \\ & & 4 & & 9 & & 1 & 0 & 6 & & 8 & \end{array}$ $\begin{array}{lcccccccccccc}\text { Preta } & 681 & 13,2 & 777 & 13,0 & 1.00 & 15,1 & 1.24 & 13,4 & 1.758 & 11,9 & 5.458 & 13,1\end{array}$ $\begin{array}{lllllllllllll}\text { Amarela } & 60 & 1,17 & 64 & 1,07 & 54 & 0,82 & 105 & 1,14 & 154 & 1,05 & 437 & 1,05\end{array}$ $\begin{array}{lcccccccccccc}\text { Parda } & 3.38 & 65,8 & 4.02 & 67,6 & 4.31 & 65,4 & 6.23 & 67,7 & 10.08 & 68,5 & 28.04 & 67,4\end{array}$ $\begin{array}{lllllllllllll}\text { ndígena } & 25 & 0,49 & 27 & 0,45 & 36 & 0,55 & 38 & 0,41 & 54 & 0,37 & 180 & 0,43\end{array}$ $\begin{array}{lllllllllllll}\text { gnorada } & 439 & 8,54 & 444 & 7,45 & 501 & 7,60 & 589 & 6,40 & 1.054 & 7,17 & 3.027 & 7,28\end{array}$ dade 


\section{ciência
plurâl}

\begin{tabular}{|c|c|c|c|c|c|c|c|c|c|c|c|c|}
\hline \multicolumn{13}{|l|}{ gestacional } \\
\hline $1^{\circ}$ & & 19,2 & 1.28 & 21,6 & 1.66 & 25,1 & 2.53 & 27,5 & \multirow{2}{*}{3.804} & 25,8 & 10.27 & \multirow{2}{*}{24,} \\
\hline Trimestre & 9 & 7 & 8 & 1 & 0 & 8 & 6 & 3 & & 7 & 8 & \\
\hline $2^{\circ}$ & 1.87 & 36,4 & 2.22 & 37,2 & 2.40 & 36,5 & 3.16 & 34,3 & \multirow{2}{*}{4.014} & 27,3 & 13.68 & 32,8 \\
\hline Trimestre & 1 & 2 & 1 & 7 & 8 & 3 & 6 & 7 & & 0 & 0 & 8 \\
\hline $3^{\circ}$ & 1.85 & 36,0 & 2.10 & 35,3 & 2.11 & 32,1 & 2.97 & 32,3 & \multirow{2}{*}{5.873} & 39,9 & 14.92 & 35,8 \\
\hline Trimestre & 2 & 5 & 7 & 5 & 9 & 5 & 5 & 0 & & 4 & 6 & 8 \\
\hline Ignorada & 424 & 8,26 & 344 & 5,77 & 405 & 6,14 & 534 & 5,80 & 1.014 & 6,89 & 2.721 & 6,54 \\
\hline \multicolumn{13}{|l|}{$\begin{array}{l}\text { Classificaçã } \\
\text { o clínica }\end{array}$} \\
\hline Sífilis & 1.85 & 36,1 & 2.05 & 34,5 & 2.17 & 32,9 & 2.93 & 31,8 & \multirow{2}{*}{4.241} & 28,8 & 13.26 & 31,8 \\
\hline Primária & 7 & 5 & 8 & 3 & 4 & 8 & 2 & 3 & & 4 & 2 & 8 \\
\hline $\begin{array}{c}\text { Sífilis } \\
\text { Secundária }\end{array}$ & 323 & 6,29 & 351 & 5,89 & 448 & 6,8 & 546 & 5,93 & 952 & 6,47 & 2.620 & 6,3 \\
\hline Sífilis & 61 & 11,9 & 728 & 12,2 & 058 & 14,5 & 1.26 & 13,7 & & 11,7 & & 12,7 \\
\hline Terciária & 015 & 7 & 728 & 1 & 958 & 3 & 6 & 4 & .733 & 9 & 300 & 4 \\
\hline Sífilis & 846 & 16,4 & 1.08 & 18,2 & 1.08 & 16,4 & 1.87 & 20,3 & 3733 & 25,3 & 8621 & 20,7 \\
\hline Latente & 840 & 7 & 8 & 6 & 3 & 3 & 1 & 1 & 3.733 & 9 & 8.021 & 2 \\
\hline Jonorado & 1.49 & 29,1 & 1.73 & 29,1 & 1.92 & 29,2 & 2.59 & 28,1 & 4046 & 27,5 & 11.80 & 28,3 \\
\hline Ignorado & 6 & 2 & 5 & 1 & 9 & 6 & 6 & 8 & 4.046 & 1 & 2 & 7 \\
\hline
\end{tabular}

\section{Discussão}

Neste estudo, observou-se aumento crescente dos casos de SG no Nordeste em todo o período investigado. Entre 2017 e 2018, embora tenha havido aumento de notificações de SG em todas as regiões brasileiras, a região Nordeste apresentou um incremento de 59,6\% nos casos novos de SG, o qual pode ter relação com a alteração do critério de definição de casos a partir de outubro de 20171. Diversos fatores podem estar contribuindo para esse aumento das notificações de sífilis em gestantes, como redução de subnotificação, realização do pré-natal pelas gestantes e o diagnóstico efetivo, bem como a rede cegonha e a utilização de testes rápidos no prénatal 6 .

Observou-se ainda aumento na taxa de detecção por 1.000 nascidos vivos. Esse indicador demonstra uma fragilidade na assistência pré-natal, a qual pode apresentar ificuldades de acesso aos serviços, bem como, problemas na abordagem, busca ativa ratamento das gestantes e seus parceiros. Entende-se, portanto, que é necessário 
ampliar o acesso, melhorar a assistência pré-natal, disponibilizar diagnóstico precoce e iniciar o tratamento em tempo hábil a fim de eliminar a SC7.

A região Nordeste apresentou no período analisado, 41.605 casos, número de notificações superior às regiões Sul, Norte e Centro-Oeste, com, respectivamente, 33.798, 20.055 e 16.663 notificações de sífilis em gestantes. No entanto, a região Sudeste registrou número superior, tendo em vista que foram notificados 97.871 $\operatorname{casos}^{8}$. Referente a isso, estudo revela que, especificamente nas regiões Nordeste e Sudeste, há limitações na atenção primária quanto ao acesso ao tratamento de SG em pelo menos $50 \%$ dos municípios, o que indica um importante fator para a perpetuação da cadeia de transmissão ${ }^{9}$.

Os Estados nordestinos que mais apresentaram casos durante o período foram Bahia, Pernambuco, Ceará e Maranhão, dentre os quais a Bahia apresentou o maior número de notificações em todos os anos estudados. Entretanto, é importante pontuar que existe subnotificação de casos, tendo em vista que estudo, realizado no Rio Grande do Norte, aponta que há maior possibilidade de subnotificações em municípios que possuem menor disponibilidade de recursos ${ }^{10}$, os quais são bastante comuns na região analisada. Desta forma, menores taxas de detecção apresentadas por alguns Estados não significam um controle efetivo da SG, visto que, entre municípios de diferentes Estados, muitas vezes há grande discrepância econômica e, consequentemente, de recursos que garantam a efetividade de diagnóstico e notificação da SG e SC. Consoante a isso, em estudo ecológico, observou-se que a incidência de SG em municípios com maior oferta de teste rápido foi até 1,63 vezes maior do que em municípios com menor oferta9.

Durante o período, verificou-se que houve aumento na taxa de detecção da SG ha região Nordeste, em que passou de 6,2, em 2014, para 18 por mil nascidos vivos, em 2018. No ano de 2018, a região Nordeste apresentou taxa de detecção menor que das outras regiões, as quais apresentaram taxas de SG por mil NV de: 18,1 (Norte),

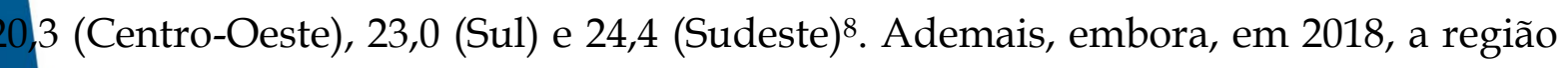
Nordeste tenha apresentado, comparada aos anos anteriores, taxa de detecção 
elevada, essa taxa foi menor que a brasileira, tendo em vista que a detecção brasileira foi de 21,4 casos de sífilis em gestantes/1.000 NV1.

No que se refere ao perfil sociodemográfico materno, estudos apontam que gestantes de raça não branca ${ }^{11-12}$, ou seja, mulheres autodeclaradas pretas ou pardas, com baixa escolaridade ${ }^{12-14}$ e na faixa etária entre 20 e $29 \operatorname{anos}^{6,14}$, são mais predominantes nos casos de sífilis, o que corrobora com os achados deste estudo. Além disso, observa-se que a sífilis afeta indivíduos com maiores vulnerabilidades sociais ${ }^{6,12}$, mas torna-se importante salientar que ela não é uma doença exclusiva das populações mais carentes ${ }^{12}$.

No que se refere à predominância de mulheres na faixa etária entre 20 e 29 anos, isso pode estar relacionado ao fato de ser a fase sexual mais intensa ${ }^{6}$. Por sua vez, a baixa escolaridade está relacionada à condição socioeconômica menos favorecida, o que compromete o acesso à assistência à saúde de qualidade ${ }^{12}$. Além disso, configura-se como fator limitante para o entendimento da importância da prevenção, sendo, portanto, considerada um fator de risco para as infecções sexualmente transmissíveis, dificultando a cessação da cadeia de transmissão ${ }^{15-16}$.

Verificou-se que os diagnósticos de sífilis na gestação foram realizados, sobretudo no $3^{\circ}$ e $2^{\circ}$ trimestres gestacionais, assim como em estudo, realizado em Palmas-TO12, o que pode estar relacionado à procura tardia à assistência pré-natal, bem como à fragilidade do rastreio de gestantes e da atenção pré-natal prestada ${ }^{15-16}$, tendo em vista que se trata de doença de detecção e tratamento simples e efetivo ${ }^{17}$. Esse achado denota a necessidade de melhorias das ações de saúde voltadas, sobretudo, pata o diagnóstico precocé ${ }^{6}$

Sabe-se que quando ocorre um pré-natal de qualidade, deve-se solicitar, no primeiro trimestre de gestação, o teste rápido para sífilis ou VDRL/RPR, ocorrendo, assim, uma captação precoce da sífilis ${ }^{18}$. Por isso, considera-se fundamental que os profissionais estejam preparados para detectar de forma rápida a sífilis nas gestantes fertar tratamento adequado tanto à gestante, quanto ao seu parceiro ${ }^{15}$, pois sabe-se a assistência de qualidade, com ações de promoção da saúde voltadas à saúde 
reprodutiva e sexual são essenciais para a prevenção de danos para a saúde materna e infantil12.

Neste estudo, segundo a classificação clínica, a maioria das gestantes foi notificada como sífilis primária, assim como em outro estudo, realizado em CaxiasMA ${ }^{16}$. Estudos apontam que o predomínio da classificação clínica, como sífilis primária, pode estar relacionado à falta de conhecimento dos profissionais de saúde sobre as fases da doença, ou seja, a possibilidade de classificação inadequada12,15-16, tendo em vista que a sífilis dificilmente é diagnosticada, durante o rastreio, na fase primária ${ }^{16}$, sendo, portanto, mais comum a fase latente ${ }^{3}$. Tal fato pode oportunizar tratamentos impróprios ${ }^{15}$. Por isso, considera-se relevante ressaltar que, na impossibilidade de estabelecimento da evolução clínica da doença, a classificação deve ser "sífilis latente de duração ignorada"12.

Ressalta-se, portanto, a importância de aperfeiçoar a competência dos profissionais de saúde para a identificação das manifestações clínicas e classificação dos estágios da doença, bem como para a interpretação dos exames e testes realizados, pois são mecanismos que auxiliam o controle do agravo, possibilitando definir o diagnóstico e monitorar a resposta terapêutica ${ }^{3}$.

\section{Conclusões}

Este estudo demonstrou que, no período estudado, os casos de sífilis em gestantes cresceram gradativamente, indicando a necessidade de ações de saúde mais efetivas, sobretudo, pelos profissionais da atenção primária. Assim, considerase fundamental que os profissionais de saúde da atenção primária sejam capacitados para intervir de maneira precoce tanto no rastreio, como no tratamento adequado das gestantes e seus parceiros a fim de minimizar os casos de sífilis gestacional e, consequentemente, de sífilis congênita. 


\section{Referências}

1. Ministério da Saúde (BR). Boletim Epidemiológico: Sífilis [Internet]. Brasília: Ministério da Saúde; 2019. [Acesso 07 Julho 2020]. Disponível em: http://www.aids.gov.br/pt-br/pub/2019/boletim-epidemiologico-sifilis-2019

2. Cerqueira LRP, Monteiro DLM, Taquette SR, Rodrigues NCP, Trajano AJB, Souza FM et al. The magnitude of syphilis: from prevalence to vertical transmission. Rev. Inst. Med. trop. S. Paulo. 2017; 59: e78. Disponível em: http://dx.doi.org/10.1590/s1678-9946201759078

3. Ministério da Saúde (BR). Protocolo clínico e diretrizes terapêuticas para prevenção da transmissão vertical do HIV, Sífilis e Hepatites virais [Internet]. Brasília: Ministério da Saúde; 2019. [Acesso 06 Janeiro 2021]. Disponível em: http://www.aids.gov.br/system/tdf/pub/2016/57801/miolo_pcdt_tv_08_2019.pdf ?file $=1 \&$ type $=$ node\&id $=57801 \&$ force $=1$

4. Gomez GB, Kamb ML, Newman LM, Mark J, Broutet N, Hawkes SJ. Sífilis materna não tratada e resultados adversos da gravidez: uma revisão sistemática e metanálise. Bull World Health Organ. 2013; 91: 217-26. Disponível em: https://dx.doi.org/10.2471\%2FBLT.12.107623

5. Silva AM, Bois F, Duro E. Factores asociados con falla en el diagnostico y tratamiento de sífilis materna. Medicina Infantil. 2016; 23(4): 293-298. Disponível em: https://www.medicinainfantil.org.ar/images/stories/volumen/2016/xxiii_4_293.p df

6. Maschio-Lima T, Machado ILL, Siqueira JPZ, Almeida MTG. Perfil epidemiológico de pacientes com sífilis congênita e gestacional em um município do Estado de São Paulo, Brasil. Rev. Bras. Saude Mater. Infant. 2019; 19(4): 865-872. Disponível em: https:/ / doi.org/10.1590/1806-93042019000400007

7. Silva PS, Vieira CSA, Gomes LMX, Barbosa TLA. Gestational and congenital syphilis in a municipality in Brazil between 2014 and 2018. DST j. bras. doenças sex. transm. 2020; 31(4): 112-117. Disponível em: https:// doi.org/10.5327/DST-21778264-201931402

8. Ministério da Saúde (BR) [homepage na internet]. Indicadores e dados básicos da sífilis nos municípios brasileiros [Acesso 08 setembro 2020]. 2020. Disponível em: http://indicadoressifilis.aids.gov.br/

Figueiredo DCMM, Figueiredo AM, Souza TKB, Tavares G, Vianna RPT. Relação tre oferta de diagnóstico e tratamento da sífilis na atenção básica sobre a incidência sífilis gestacional e congênita. Cad. Saúde Pública. 2020; 36(3): e00074519.

sponível em: http:/ /dx.doi.org/10.1590/0102-311x00074519 
10. Carvalho IS, Brito RS. Sífilis congênita no Rio Grande do Norte: estudo descritivo do período 2007-2010. Epidemiol Serv Saúde. 2014; 23(2): 287-94. Disponível em: https:/ / doi.org/10.5123/S1679-49742014000200010

11. Lafetá KRG, Martelli Júnior H, Silveira MF, Paranaíba LMR. Sífilis materna e congênita, subnotificação e difícil controle. Rev. bras. epidemiol. 2016; 19(1): 63-74. Disponível em: http:/ / dx.doi.org/10.1590/1980-5497201600010006

12. Padovani C, Oliveira RR, Pelloso SM. Syphilis in during pregnancy: association of maternal and perinatal characteristics in a region of southern Brazil. Rev. Latino-Am. Enfermagem. 2018; 26: e3019. Disponível em: http://dx.doi.org/10.1590/15188345.2305.3019

13. Cabral BTV, Dantas JC, Silva JA, Oliveira DA. Sífilis em gestante e sífilis congênita: um estudo retrospectivo. Revista Ciência Plural. 2017; 3(3): 32-44. Disponível em: https:// periodicos.ufrn.br/rcp/article/view/13145

14. Pereira LC, Santos VF, Albuquerque ACD, Lages CM, Cunha KJB, Silva DP. Perfil epidemiológico de los casos de sífilis en embarazadas en una maternidad pública. Cultura de los cuidados. 2019; (54): 396-400. Disponible en: http:// ciberindex.com/c/cc/54396cc

15. Cavalcante PAM, Pereira RBL, Castro JGD. Sífilis gestacional e congênita em Palmas, Tocantins, 2007-2014. Epidemiol. Serv. Saúde. 2017; 26(2): 255-264. Disponível em: https:// doi.org/10.5123/s1679-49742017000200003

16. Conceição HN, Câmara JT, Pereira BM. Análise epidemiológica e espacial dos casos de sífilis gestacional e congênita. Saúde debate. 2019; 43(123): 1145-1158. Disponível em: https://doi.org/10.1590/0103-1104201912313

17. Almeida PD, Araujo Filho ACA, Araújo AKL, Carvalho ML, Silva MGP, Araújo TME. Análise epidemiológica da sífilis congênita no Piauí. R. Interd. 2015; 8(1): 62-70. Disponível em:

https:/ / revistainterdisciplinar.uninovafapi.edu.br/index.php/revinter/article/view $/ 453$

18. Machado I, Silva VAN, Pereira RMS, Guidoreni CG, Gomes MP. Diagnóstico e tratamento de sífilis durante a gestação: Desafio para enfermeiras?. Rev. Saúde e Pesquisa. 2018; 11(2): 249-255. Disponível em: https:/ / doi.org/10.17765/21769206.2018v11n2p249-255 